\title{
IMPLEMENTATION OF THE CAN BUS IN THE VEHICLE BASED ON
}

\section{ARM 7}

\author{
Vijaya D. Kokane' ${ }^{1}$ Prof. Sanjay B. Kalyankar ${ }^{2}$ \\ ${ }^{1}$ Department of Electronics \& Telecommunication, Deogiri Institute of Engg. \& Management Studies, Aurangabad \\ ${ }^{2}$ Department of Electronics \& Telecommunication, Deogiri Institute of Engg. \& Management Studies, Aurangabad
}

\begin{abstract}
A controller area network (CAN) is mostly used to the many high level industrial protocol, due to it reducing wiring harness, tremendous flexibility, high performance, low cost. This paper represent the CAN used in the motor car, which show different parameter by using different sensors, the different types of sensors to measure the different parameter like temperature, humidity ,gas detection \& fuel level based on ARM (LPC 2129). Microcontroller ARM (LPC 2129) has inbuilt CAN controller.
\end{abstract}

Keywords: CAN bus, ARM7 (LPC2129) , MCP2551 ,MQ6 LPG gas detector, SY-HS 220 humidity detector, LM 35 temperature sensor.

\section{INTRODUCTION}

In the starting days of the developing the technology, the use of Electronics Control Units (ECUs) increases rapidly . To exchange the information between ECUs, it is necessary to connect them. This is usually done by the discrete interconnection of the different system (i.e. point to point cabling). As the use of ECUs is increases the length of cable also grown to such an extent that of several miles . This produces problem concerning material cost , production cost \& reliability .

To solve these problem we implement the CAN Bus \& connect all the ECUs through that CAN bus . The CAN bus replaces all point to point cabling through a single bus . This is done by adding some CAN-specific hardware to each control unit that provides "rules" or "protocol" for transmitting \& receiving the information through CAN bus . CAN bus is a simple two wire protocol. It is an asynchronous serial communication protocol . It is mostly used in automobile Industry due to it's real time performance $\&$ reliability . It is half duplex system $\&$ has data rate up to $1 \mathrm{Mbps}$. It provide high level of security.

This research paper design the implementation of the CAN bus in vehicle based on ARM 7 i.e. LPC 2129 which contain inbuilt CAN controller . The MCP2551 is a highspeed CAN \& mostly used as a voltage converter .It convert general voltage level to CAN voltage level at the transmitter \& CAN voltage level to general voltage level at the receiver - MQ6-LPG is a gas sensor \& is used to detect the gas leakage . It is mostly used to detecting LPG , iso-butane, propane, LNG combustible gases. SY-HS 220 is a humidity sensor \& used to measure the amount of water molecule present in the air. this sensor then convert humidity into output voltage. LM 35 series are temperature sensor, whose output voltage is linearly proportional to the temperature in (celcius) . LM 35 , user is not require to subtract a large constant voltage from it's output to obtain convenient scaling. The most commonly used Character based LCDs to display the output .

\section{LITERATURE SURVEY}

The Robert Bosch GmbH started the development of Controller Area Network (CAN) in 1983 \& officially released this protocol in 1986 at the Society of Automotive Engineers (SAE) Congress in betroit, Michigan . The first CAN chip come on in the market in 1987 , which is produced by Intel \& Philips . In 1991 Bosch published the CAN 2.0 specification . Bosch originally develop the CAN controller for used in Vehicle network in 1985.

As the technology is developing the use of Electronics control units (ECUs) also increased in the vehicle. In the starting of the developing days the different ECUs connected by discrete interconnection (i.e. point to point cabling). This cable networking having more complexity in the system \& high cost effective. The solution to this problem was introduced by using Multiplex communication to reduced complexity between different ECUs . But the Multiplex communication has not meet the real time communication requirement . In the early, 1980s there was no suitable real-time control system . The CAN protocol Internationally standardized as ISO $11898-1$ to solve all these problem .

The higher level protocol of the CAN bus was developed in 1991 , CAN kingdom was introduced by Kvaser . The higher level protocol are Device-Net, open CAN, J1939 . In 1992 CAN Bus is used in passenger bus by Merecdes Benz . 


\section{Block diagram of Proposed System}

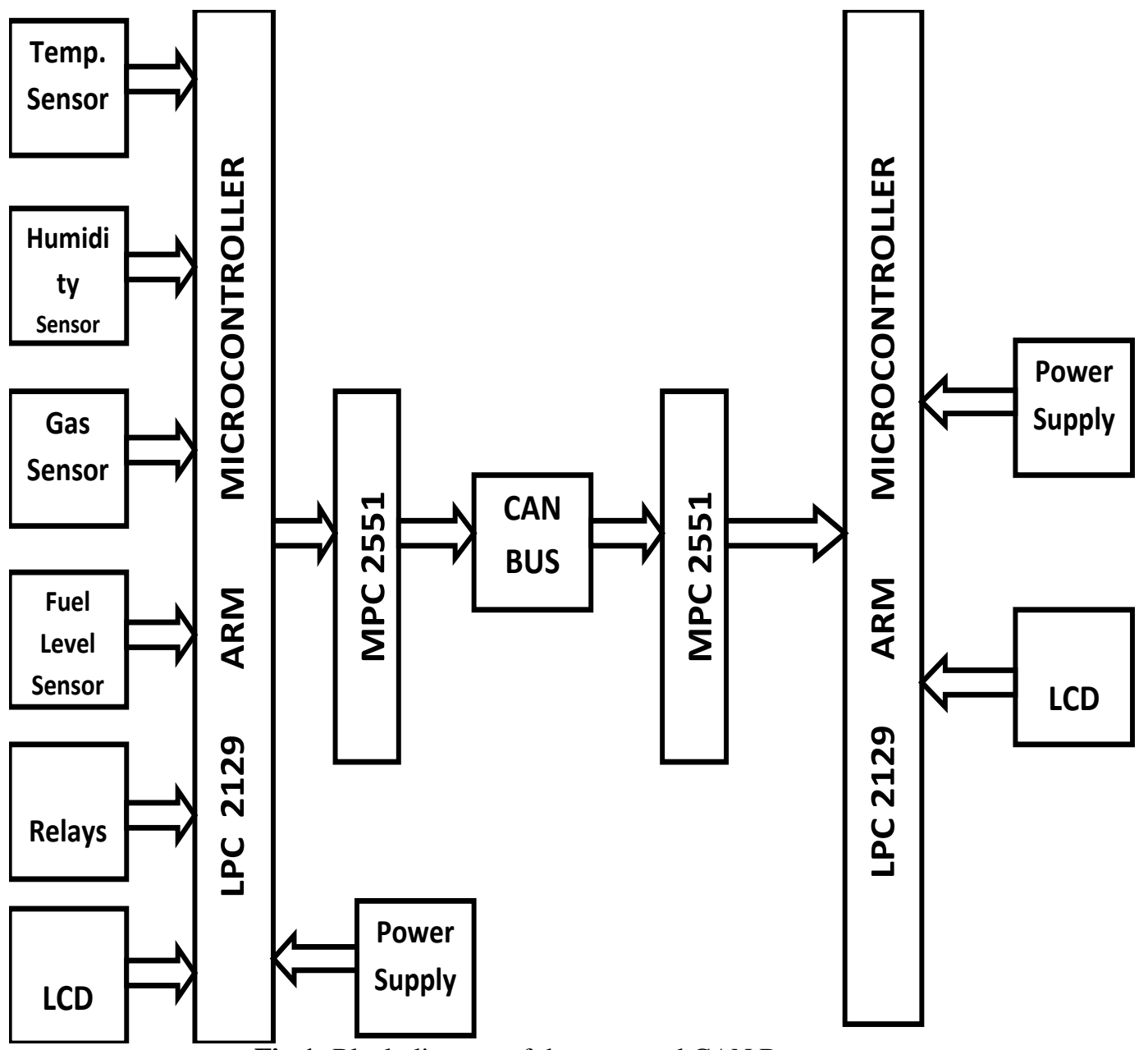

Fig 1: Block diagram of the proposed CAN Bus system

Fig. shows the block diagram of development of the CAN Bus system, which contain transmitter \& receiver section . In the transmitter section different type of the sensor are used to measure the parameter like for measurement of the temperature use temp. sensor LM35 ,or humidity measurement use SY-HS-220 humidity sensor ,for gas detection use MQ6 LPG gas sensor ,for fuel level indication use the circuit . 7805 IC is used as power supply having two fixed voltage i.e. $3.3 \mathrm{v} \& 5 \mathrm{v}, 3.3 \mathrm{v}$ is required or microcontroller IC LPC 2129 \& 5v required to the other circuit. the microcontroller ARM7 LPC 2129 is used at both side i.e. at transmitter $\&$ receiver side to transmit $\&$ receive the data, which contain built in CAN controller .

IC MPC 2551 is used to convert the voltage level. In the CAN bus there are two level CAN H \& CAN L there is no ground level .In the CAN bus ,If CAN H - 2.5v/3.7v \& CAN L $-2.5 \mathrm{v} / 3.7 \mathrm{v}, \mathrm{CAN} \mathrm{H}-\mathrm{CAN} \mathrm{L}=2.5-2.5=0 \mathrm{v}$ consider as Logic ' 0 ' , If CAN $\mathrm{H}=4.1 \mathrm{~V} \& \mathrm{CAN} \mathrm{L}=1.5 \mathrm{~V}$ ,CAN H - CAN L = 4.1- $1.5=2.6 \quad$ consider as logic ' 1 ' ' But in another IC consider $3.3 \mathrm{v}$ is logic ' 1 ' \& $0 \mathrm{v}$ is logic ' 0 ' $\&$ also required the ground so the IC MCP 2551 is used to convert general level voltage to the $\mathrm{CAN}$ level voltage .
LCD is used to display the value. CAN Bus is used to transmit \& receive the data which is explain later.

\begin{abstract}
ARM7 - LPC 2129
ARM7 is a32 - bit microcontroller which provides high performance \& low power consumption . The ARM7 LPC 2129 is based on Reduction Instruction Set Computer (RISC) principle. The Instruction set $\&$ decode mechanism of the ARM7 is simpler than the micro programmed complex instruction set . so the reduced in the instruction set the instruction throughput is high \& impressive real tie response . It has small size $\&$ less cost processor core .
\end{abstract}

The pipeline technique are employed in this ARM so that all the processor \& memory system an be operated simultaneously . In the typical microcontroller one instruction is being executed, second is being decoded \& third instruction is being fetched from memory .

The ARM 7 processor also employ the unique architecture strategy know as THUMB . The key idea behind this THUMB strategy is the super reduced instruction set . It is used in high volume application . 
In the ARM 7 there are two instruction sets ,

1) Standard 32-bit instruction set

2) 16-bit THUMB instruction set

16-bit THUMB instruction set having twice density than standard 32-bit instruction set .

\section{Combustible Gas Sensor - Analog Out (MQ6 LPG)}

MQ6-LPG is a gas sensor \& is used to detect the gas leakage . It is mostly used to detecting LPG, iso-butane, propane, LNG combustile gases. The sensor does not trigger to the other noises like alcohol, cooking fumes \& cigarette smoke or any other noises . This is mostly design to allow microcontroller to determine when present gas level has been reached.

\section{LM35 Precision Centigrade Temperature Sensors}

LM 35 series are temperature sensor, whose output voltage is linearly proportional to the temperature in (celcius) . LM 35 , user is not require to subtract a large constant voltage from it's output to obtain convenient scaling. This is the advantage of LM 35 series over linear temperature sensor calibrated in Kelvin . To provide proper accuracy LM35 does not require any external calibration or trimming circuit . LM 35 has low output impedance, linear output \& precise inherent calibration. It can be operated on both power supply i.e. single power supply or with plus \& minus supplies .

\section{Humidity Sensor Module SY-HS-220}

The humidity sensor are used to measure the amount of water present in the air. The SY-HS 220 module is used to measure humidity $\&$ convert the relative humidity to the output voltage.

\section{CONCLUSION}

This paper describes about implementing the CAN Bus in vehicle using microcontroller ARM7 (LPC 2129) \& voltage converter IC MCP 2551which convert the voltage level .We can show here four parameter of vehicle by using CAN Bus like temperature ,humidity, gas detection $\&$ wiper rotation. This research proposes the design of implement o the CAN Bus in the vehicle to improve the data transformation speed between Electronics control devices , LPC 2129 which contain inbuilt CAN controller . MQ6-LPG is a gas sensor $\&$ is used to detect the gas leakage . It is mostly used to detecting LPG, iso-butane, propane, LNG combustible gases. The sensor does not trigger to the other noises like alcohol, cooking fumes \& cigarette smoke or any other noises. SY-HS 220 is a humidity sensor \& used to measure the amount of water molecule present in the air . this sensor then convert humidity into output voltage. The LM35 series are temperature sensors, whose output voltage is linearly proportional to the temperature . this temperature sensor is not require to subtract a large constant voltage from it's output to obtain convenient scaling. The most commonly used Character based LCDs are based to display the output .

\section{REFERENCES}

[1] Sunil Kumar Gurram and James M. Conrad Implementation of CAN bus in an autonomous AllTerrain Vehicle 2010-2011

[2] Richard A. McKinney, Malcolm J. Zapata, James M. Conrad, Thomas W. Meiswinkel and Siddharth Ahuja, Components of an Autonomous All-Terrain Vehicle, IEEE SoutheastCon, 2010

[3] https://www.sparkfun.com/datasheets/Sensors/Bio metric/MQ-6 TECHNICAL DATA. MQ-6 GAS SENSOR. FEATURES. * High sensitivity to $L P G$, iso-butane, propane.

[4] National Semiconductor October 2000 LM35 Precision Centigrade Temperature Sensors

[5] www.engineersgarage.com/articles/humiditysensor

[6] www.keil.com/dd/docs/datashts/philips/lpc2119_2 129.

[7] support.ricoh.com/bb/html/dr_ut_e/ra/model/mpc2 $1 /$ mpc21en 